\title{
Transcriptome sequencing reveals that LPS-triggered transcriptional responses in established microglia BV2 cell lines are poorly representative of primary microglia
}

Amitabh Das ${ }^{1 \dagger}$, Sun Hwa Kim² ${ }^{2 \dagger}$, Sarder Arifuzzaman ${ }^{3}$, Taeho Yoon², Jin Choul Chai², Young Seek Lee², Kyoung Sun Park', Kyoung Hwa Jung ${ }^{1 *}$ and Young Gyu Chai ${ }^{2,3^{*}}$

\begin{abstract}
Background: Microglia are resident myeloid cells in the CNS that are activated by infection, neuronal injury, and inflammation. Established BV2 microglial cell lines have been the primary in vitro models used to study neuroinflammation for more than a decade because they reduce the requirement of continuously maintaining cell preparations and animal experimentation models. However, doubt has recently been raised regarding the value of BV2 cell lines as a model system.

Methods: We used triplicate RNA sequencing (RNA-seq) to investigate the molecular signature of primary and BV2 microglial cell lines using two transcriptomic techniques: global transcriptomic biological triplicate RNA-seq and quantitative real-time PCR. We analyzed differentially expressed genes (DEGs) to identify transcription factor (TF) motifs ( -950 to +50 bp of the $5^{\prime}$ upstream promoters) and epigenetic mechanisms.

Results: Sequencing assessment and quality evaluation revealed that primary microglia have a distinct transcriptomic signature and express a unique cluster of transcripts in response to lipopolysaccharide. This microglial signature was not observed in BV2 microglial cell lines. Importantly, we observed that previously unidentified TFs (i.e., IRF2, IRF5, IRF8, STAT1, STAT2, and STAT5A) and the epigenetic regulators KDM1A, NSD3, and SETDB2 were significantly and selectively expressed in primary microglia (PM). Although transcriptomic alterations known to occur in BV2 microglial cell lines were identified in PM, we also observed several novel transcriptomic alterations in PM that are not frequently observed in BV2 microglial cell lines.
\end{abstract}

Conclusions: Collectively, these unprecedented findings demonstrate that established BV2 microglial cell lines are probably a poor representation of PM, and we establish a resource for future studies of neuroinflammation.

Keywords: Gene regulation, Innate immunity, Transcription factors, Microglia, RNA sequencing

\footnotetext{
*Correspondence: khjung2@gmail.com; ygchai@hanyang.ac.kr

${ }^{\dagger}$ Equal contributors

'Institute of Natural Science and Technology, Hanyang University, Ansan

15588, Republic of Korea

${ }^{2}$ Department of Molecular and Life Sciences, Hanyang University, Ansan

15588, Republic of Korea

Full list of author information is available at the end of the article
} 


\section{Background}

It has become increasingly evident that neuroinflammation, triggered by the activation of glial cells, plays a key role in many neurodegenerative disorders, such as Alzheimer's, Parkinson's, and Huntington's disease and multiple sclerosis $[1,2]$. Microglia, the principal resident macrophages of the brain and spinal cord, comprise 5-12\% of brain cells and act as primary effector cells. These cells play an important role in the brain's innate immunity, neuronal homeostasis, and neuroinflammatory pathologies $[2,3]$. Microglia become rapidly activated in response to infection, inflammation, or brain injury. The activated microglia release various inflammatory mediators, including tumor necrosis factor-alpha (TNF- $\alpha$ ), interleukin (IL)1B, IL6, nitric oxide (NO), reactive oxygen species (ROS), and prostaglandin E2 $\left(\mathrm{PGE}_{2}\right)$ which have been implicated in various neurodegenerative diseases $[1,4]$. However, the mechanisms that regulate microglial activation have not been completely defined.

Microglia express a broad range of pattern recognition receptors in the toll-like receptor (TLR) family to detect microbial intruders and brain damage [5]. Among these, bacterial cell wall endotoxin lipopolysaccharide (LPS), the ligand for toll-like receptor 4 (TLR4), is one of the most potent stimuli that induces microglial activation. LPS activates microglia, leading to the release of cytokines and a host of neurotoxic factors that induce neuronal death [6, 7]. Most research on microglial TLR signaling has been performed in vitro, usually by using cell lines, such as N9 [8] and BV2 cell lines [9]. During the past decade, in our monitoring of the PubMed search "BV2 and neuroinflammation," we have witnessed an explosion of work aimed at understanding the role of microglia in neurodegenerative disorders. Doubt has been raised regarding the value of the BV2 cell lines as a model system. BV2 cell lines were originally derived from v-raf/v-myc-immortalized murine neonatal microglia, and this cell line is the most frequently used alternative to using primary microglia (PM). For example, previous studies reported that in the presence of LPS, transcriptomic and proteomic analyses of BV2 cell lines revealed similarities with PM [10]. Recently, other laboratories have reported that BV2 cell lines exhibit many similarities with PM and in vivo models in studies of Huntington's disease [11]. In addition, BV2 cell lines are used in the pharmaceutical industry [12]. However, immortalization causes these cells to be different from PM in culture or in the brain [13]. For instance, previous studies have demonstrated that after exposure to macrophage colony-stimulating factor (MCSF) and transforming growth factor beta 1 (TGF- 31 ), adult PM showed a unique molecular expression profile that was different from the profile in BV2 cell lines [14]. Furthermore, immortalization through transfection with oncogenes renders these cells some ways different from PM, in terms of morphology, proliferation, and adhesion $[13,15]$. Thus, it remains unclear how closely established cell lines resemble $\mathrm{PM}$ in a comprehensive characterization of phenotypic activation.

Although a few studies have compared the effects of LPS in vitro and in vivo $[10,16]$, a comprehensive and comparative transcriptional profile of responses to this stimulus has not been performed using the RNA sequencing (RNA-seq) to compare results between established cell lines and PM. To analyze the transcriptomes of tissues and cells, several approaches have already been developed. Among the various available technologies, microarrays are very useful, but they provide only a semi-quantitative assessment of the transcriptome. In contrast, RNA-seq platforms are quantitative, and they provide unbiased profiles, a snapshot of the transcriptome of cells at a specific time point, and the ability to identify novel transcribed regions, unlike microarrays, and they can therefore be extremely accurate if a sufficient level of coverage is obtained $[17,18]$. Using this approach, we analyzed the unbiased quantitative transcriptome of PM and compared it to that of cell lines. The outcome of these studies allowed us to identify a common and unique PM transcriptional signature distinct from the BV2 cell lines. To the best of our knowledge, our dataset is the first quantitative transcriptomic analysis to compare BV2 cell lines and PM.

\section{Methods}

\section{Cell culture, stimulation, and morphological analysis of BV2 cell lines and PM}

Mouse microglial BV2 cell lines were grown in highglucose Dulbecco's modified Eagle's medium (DMEM) supplemented with $10 \%$ fetal bovine serum (FBS) (cata$\log$ \# 26140; Gibco, Waltham, MA), $100 \mathrm{IU} / \mathrm{ml}$ penicillin, and $10 \mu \mathrm{g} / \mathrm{ml}$ streptomycin (catalog \# 15140; Invitrogen, Waltham, MA). The cells were maintained in a humidified incubator with $95 \%$ air and a $5 \% \mathrm{CO}_{2}$ atmosphere at $37{ }^{\circ} \mathrm{C}$. Medium containing the appropriate agents was replaced every other day. PM were isolated from 3-day-old ICR mice as previously described [19] with minor modifications. All experimental protocols were performed in accordance with the Institutional Animal Care and Use Committee (IACUC) guidelines and approved by the IACUC committee of Hanyang University (HY-IACUC-2014-0164A and HYIACUC-2015-0075). Briefly, whole brains of neonatal mice were dissected out of the skull, and blood vessels and meninges were carefully removed. Then, the tissues from whole brains obtained from 12 mice were pooled together, finely minced, and digested using a Neural Tissue Dissociation Kit-Postnatal Neurons (Miltenyi Biotec, Germany, 130-094-802). Next, the digested cells 
were passed through a 70- $\mu \mathrm{m}$ nylon cell strainer (BD Bioscience, Franklin Lakes, NJ) and seeded in poly-Llysine-coated T-75 flasks in DMEM/nutrient mixture F-12 (DMEM/F12, 1:1) containing $20 \%$ FBS (catalog \# 26140; Gibco, Waltham, MA), $100 \mathrm{IU} / \mathrm{ml}$ penicillin, and $10 \mu \mathrm{g} / \mathrm{ml}$ streptomycin (catalog \# 15140) obtained from Invitrogen (Waltham, MA). The cells were maintained in a humidified incubator with a $95 \%$ air $/ 5 \% \mathrm{CO}_{2}$ atmosphere at $37{ }^{\circ} \mathrm{C}$. The medium was changed every 2 3 days. After 2 weeks in culture, the mixed glial cell cultures were shaken at $150 \mathrm{rpm}$ at $37^{\circ} \mathrm{C}$ for $45 \mathrm{~min}$, and the glial cell suspensions were collected from each flask and seeded on poly-L-lysine-coated cell culture plates. Microglial cells were sub-plated and used for further experiments. The morphology of BV2 cell lines and PM at $4 \mathrm{~h}$ with and without (control) treatment with LPS was analyzed for each independent experiment (Additional file 1: Figure S1A). More than 95 and $92 \%$ of cells obtained were BV2 cell lines and PM microglia, respectively, as quantified by CD11b (rat monoclonal immunoglobulin G2b (IgG2b), clone: M1/70.15.11.5, Miltenyi Biotec Germany) FACS analysis (Additional file 1: Figure S1B). The cells were treated with LPS $(10 \mathrm{ng} / \mathrm{ml})$ and incubated for 2 and $4 \mathrm{~h}$ under BV2 cell lines and PM culture conditions, respectively. LPS (L6529; strain 055:B5) was purchased from Sigma-Aldrich, St. Louis, MO.

\section{Total RNA isolation and cDNA library preparation for transcriptome sequencing (RNA-seq)}

Total RNA was extracted using RNAiso Plus (Takara Bio Inc., Shiga, Japan) and a QIAGEN RNeasy ${ }^{\circ}$ Mini kit (QIAGEN, Hilden, Germany). BV2 cell lines or PM cells were completely lysed using RNAiso Plus, and then, $200 \mu \mathrm{l}$ of chloroform was added. The tubes were then inverted for $5 \mathrm{~min}$. The mixture was centrifuged at $12,000 \times g$ for $15 \mathrm{~min}$ at $4{ }^{\circ} \mathrm{C}$, and the upper phase was placed into a new tube. A $600 \mu \mathrm{l}$ volume of $70 \%$ ethanol was added, and the mixture was applied to an RNeasy mini column. The column was washed with wash buffer. To elute the RNA, RNase-free water $(30 \mu \mathrm{l})$ was added directly onto the RNase mini column, which was then centrifuged at $12,000 \times g$ for $3 \mathrm{~min}$ at $4{ }^{\circ} \mathrm{C}$. To deplete ribosomal RNA (rRNA) from the total RNA preparations, a RiboMinus Eukaryote kit (Life Technologies, Carlsbad, CA) was used according to the manufacturer's instructions. RNA libraries were created using a NEB$\mathrm{Next}^{\circ}$ Ultra $^{\mathrm{Tm}}$ directional RNA library preparation kit for Illumina ${ }^{\circ}$ (New England BioLabs, Ipswich, MA). The obtained rRNA-depleted total RNA was fragmented into small pieces using divalent cations at elevated temperatures. First-strand complementary DNA (cDNA) was synthesized using reverse transcriptase and random primers, and second-strand cDNA synthesis was then performed using DNA polymerase I and RNase $\mathrm{H}$. The
cDNA fragments were processed using an end-repair reaction after the addition of a single "A" base, followed by adapter ligation. These products were purified and amplified using PCR to generate the final cDNA library. The cDNA fragments were sequenced using an Illumina HiSeq2000. Biological triplicate RNA sequencing was performed on 18 independent RNA samples of BV2 cell lines and PM cells, i.e., control BV2 (3 samples), BV2 LPS $2 \mathrm{~h}$ (3 samples), BV2 LPS $4 \mathrm{~h}$ (3 samples), control PM (3 samples), PM LPS $2 \mathrm{~h}$ (3 samples), and PM LPS $4 \mathrm{~h}$ (3 samples). We selected the 2- and 4-h time point for whole-genome transcriptional profiling based on previous PCR array data that showed that the optimal induction of immune response genes occurs at this time point when microglia are activated using LPS [16, 20, 21].

Differentially expressed gene analysis using RNA-seq data FASTQ files from RNA-seq experiments were clipped and trimmed of adapters, and the low-quality reads were removed by the Trimmomatic [22]. Quality-controlled FASTQ files were aligned to mus musculus UCSC mm10 reference genome sequence using the STAR (version 2.5.1) aligner software [23] with three mismatches. To measure differential gene expression, DESeq2 [24] with the default parameters was used. A subset of conditionspecific expression was defined as showing a $\log _{2}$ fold change $\geq 1.5$ and $P \leq 0.01$ in expression between controls and LPS-treated samples. To further characterize the BV2 cell lines and PM cells, we selected different immunoregulatory (cytokines, chemokines, interferon response genes, etc.) genes based on functionally related according to current knowledge. The RNA-seq experiments were visualized using HOMER (version 4.7) [25] after custom tracks were prepared for the UCSC Genome Browser (http://genome.ucsc.edu/). The acquired data were deposited in the Gene Expression Omnibus database under dataset accession nos. GSE79898 and GSE80304.

\section{Quantitative real-time RT-PCR}

The reverse transcription of the RNA samples was performed as previously described [26] using $2 \mu \mathrm{g}$ of total RNA, $1 \mu$ lof oligo (dT) primer (per reaction), and a PrimeScript 1st strand cDNA Synthesis Kit (Takara Bio Inc., Shiga, Japan). The oligo (dT) primer and RNA templates were mixed and denatured at $65^{\circ} \mathrm{C}$ for $5 \mathrm{~min}$ and then cooled for $2 \mathrm{~min}$ on ice. PrimeScript buffer $(5 \times)$, RTase, and RNase inhibitor were added to the cooled template mixture and incubated for $1 \mathrm{~h}$ at $50{ }^{\circ} \mathrm{C}$ before an enzyme inactivation step was performed at $70{ }^{\circ} \mathrm{C}$ for $15 \mathrm{~min}$. Quantitative real-time RT-PCR (qRTPCR) was performed using SYBR Green PCR Master Mix (Takara Bio Inc., Shiga, Japan) and a 7500 Fast RealTime PCR System (Applied Biosystems, Waltham, MA). Glyceraldehyde-3-phosphate dehydrogenase (GAPDH) 
was used as an internal control. Complementary DNA samples were diluted 1.5-fold, and qRT-PCT was performed using an ABI-7500 Real-Time PCR System (Applied Biosystems, Waltham, MA) with SYBR Premix Ex-Taq II (Takara Bio Inc., Shiga, Japan) according to the manufacturer's instructions. The reactions were performed in a total volume of $20 \mu \mathrm{l}$ that contained $0.4 \mathrm{mM}$ of each primer (Table 1). Each PCR series included a notemplate control that contained water instead of cDNA and a reverse transcriptase-negative control for each gene. Triplicate measurements were performed for all reactions. Different samples were evaluated using 96-well plates in the gene expression experiments, and all samples were analyzed on a single plate for the endogenous control experiments. The results were analyzed using the critical threshold $\left(\Delta C_{\mathrm{T}}\right)$ and comparative critical threshold $\left(\Delta \Delta C_{\mathrm{T}}\right)$ methods in the ABI-7500 software program with the Norm finder and geNorm-plus algorithms. The primers were designed using Primer Express software (Applied Biosystems, Waltham, MA).

\section{Functional annotation}

To functionally annotate the most significant genes, gene ontology analysis was performed using DAVID (Database for Annotation, Visualization and Integrated Discovery), version 6.8 [27]. Gene ontology was analyzed using a modified Fisher's exact $P$ value in the DAVID program. $P$ values less than 0.001 were considered to be greatly enriched in the annotation category.

\section{Canonical pathway analysis of datasets}

An Ingenuity Pathway Analysis (IPA) (Ingenuity Systems, http://www.ingenuity.com, CA) was performed to analyze the most significant canonical pathways in the datasets as previously described [28]. The genes from datasets associated with canonical pathways in the Ingenuity Pathways Knowledge Base (IPAKB) were considered for literary analysis. The significance of the associations between datasets and canonical pathways was measured in the following manner: (1) the ratio of the number of genes from the dataset that mapped to a canonical pathway was divided by the total number of genes that mapped to the same canonical pathway and (2) Fisher's exact test for a $P$ value indicating the probability that the association could be explained by chance. After uploading the datasets, gene identifiers were mapped to corresponding gene objects, and the genes were overlaid onto a global molecular network in the IPAKB. Gene networks were algorithmically generated based on connectivity.

\section{Transcription factor-binding motif enrichment analysis}

NCBI reference sequence messenger RNA (mRNA) accession numbers were subjected to transcription factorbinding motif analysis using the web-based software Pscan [29]. The JASPAR [30] database of transcription factor (TF)-binding sequences was analyzed using enriched groups of -950 base pair (bp) sequences to $+50 \mathrm{bp}$ of the $5^{\prime}$ upstream promoters. The range -950 to +50 was selected from the range options in Pscan to obtain the best coverage for a -1000 - to +50 -bp range.

\section{Statistical analysis}

The data were analyzed using Origin Pro 8 software (Origin Lab Corporation, Northampton, MA). Each value is expressed as the mean \pm standard error of the mean (SEM). All qRT-PCR data were analyzed with SPSS 17.0 software

Table 1 List of primers used in qRT-PCR studies

\begin{tabular}{|c|c|c|}
\hline Gene designation & Forward $\left(5^{\prime} \rightarrow 3^{\prime}\right)$ & Reverse $\left(5^{\prime} \rightarrow 3^{\prime}\right)$ \\
\hline CCL4 & TTCCTGCTGTTTCTCTTACACCT & CTGTCTGCCTCTTTTGGTCAG \\
\hline$C C L 5$ & TITGCCTACCTCTCCCTCG & CGACTGCAAGATTGGAGCACT \\
\hline CCL8 & CTGGGCCAGATAAGGCTCC & CATGGGGCACTGGATATTGTT \\
\hline CXCL1 & ACTGCACCCAAACCGAAGTC & TGGGGACACCTITAAGCATCTT \\
\hline IFNB1 & AGCTCCAAGAAAGGACGAACA & GCCCTGTAGGTGAGGTTGAT \\
\hline IFIT1 & GCCTATCGCCAAGATTTAGATGA & TTCTGGATTTAACCGGACAGC \\
\hline IFIT2 & GGAGAGCAATCTGCGACAG & GCTGCCTCATTTAGACCTCTG \\
\hline IFIT3 & CCTACATAAAGCACCTAGATGGC & ATGTGATAGTAGATCCAGGCGT \\
\hline IRF1 & ATG CCA ATC ACT CGA ATG CG & TTG TAT CGG CCT GTG TGA ATG \\
\hline IRF2 & AATTCCAATACGATACCAGGGCT & GAGCGGAGCATCCTTITCCA \\
\hline STAT1 & TCACAGTGGTTCGAGCTTCAG & CGAGACATCATAGGCAGCGTG \\
\hline STAT2 & GTTACACCAGGTCTACTCACAGA & TGGTCTTCAATCCAGGTAGCC \\
\hline KDM4A & GAC CAC ACT CTG CCC ACA C & TCC TGG GGT ATT TCC AGA CA \\
\hline SETDB2 & TGGGTCTGCCACAAATGGAG & TCCAGTGTTTGCGTGTTACTC \\
\hline GAPDH & TGCGACTTCAACAGCAACTC & CTTGCTCAGTGTCCTTGCTG \\
\hline
\end{tabular}


(SPSS Inc., Chicago, IL). The data were tested using one-way ANOVA followed by Tukey's HSD post hoc test. ${ }^{*} P<0.01$ and ${ }^{* *} P<0.001$ were considered significant.

\section{Results}

Gene-induction patterns following TLR4 activation in PM

To determine the proper time course responses, we performed an expression analysis in TLR4-stimulated versus control PM. LPS $(10 \mathrm{ng} / \mathrm{ml})$ caused a transient up-regulation of key inflammatory response-related genes, peaking at 2 and $4 \mathrm{~h}$ for TNF- $\alpha$, CXCL10, IL1A, IL1B, CCL4, and CCL5 (Fig. 1a). In response to stimulation by different doses of LPS (10-100 ng/ml), BV2 cell lines and PM caused significant up-regulation of key inflammatory response-related genes at 2 and $4 \mathrm{~h}$. The fold induction in the increase of TNF- $\alpha$ and IL1B in response to different doses of LPS (10-100 $\mathrm{ng} / \mathrm{ml})$ was similar in both cell types at 2 and $4 \mathrm{~h}$ (Fig. 1b).

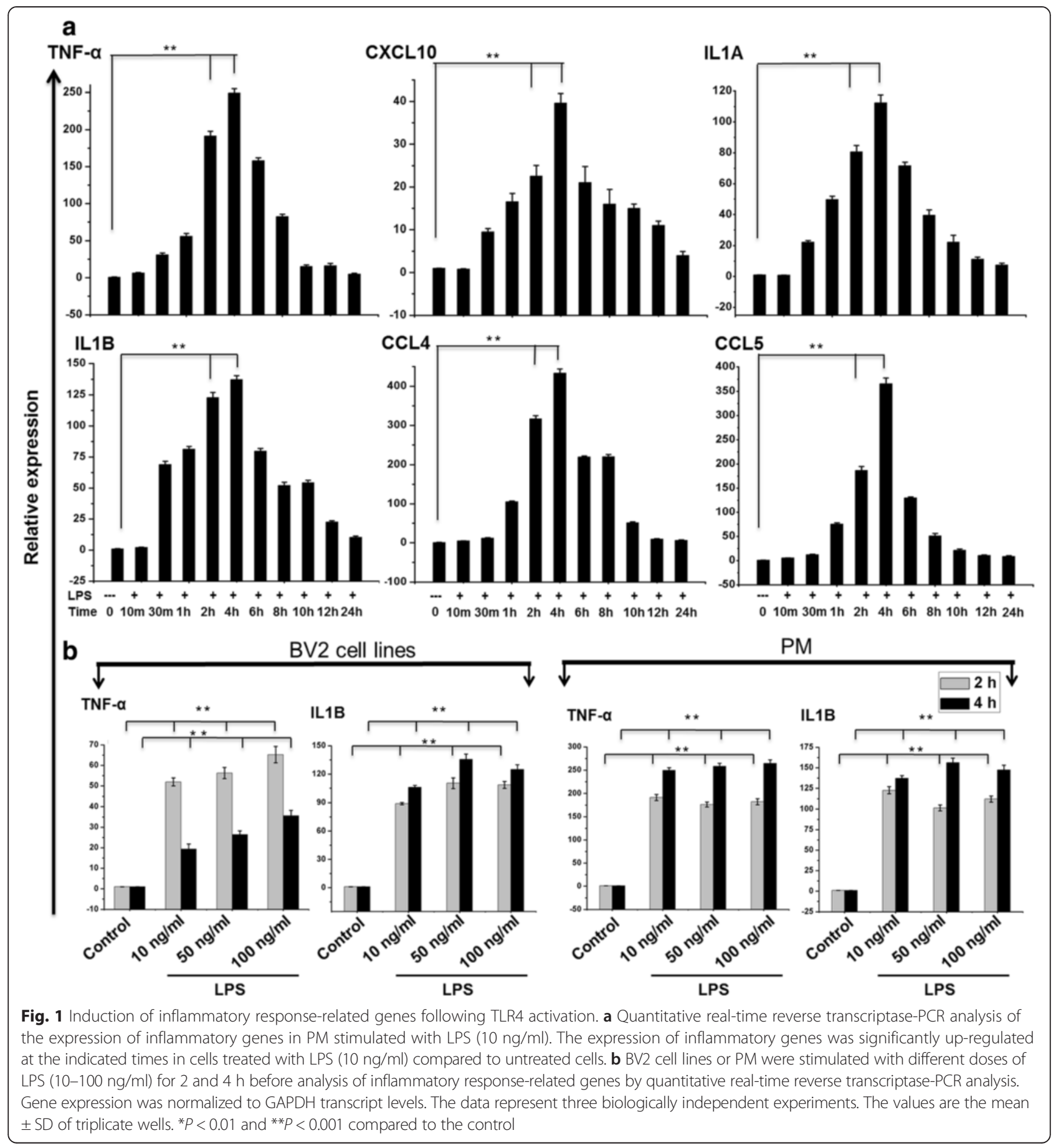


We hence used the lower doses for subsequent analyses. Notably, morphological analysis shows that at 4 h, LPS-treated BV2 cell lines and PM show similar morphology and responses as compared to control cells (Additional file 1: Figure S1A).

\section{RNA-seq transcriptional comparison between PM and BV2 cell line microglia following TLR4 activation}

To gain a comprehensive understanding of the mechanisms involved and to directly compare how TLR4 stimulation alters the transcriptomic profile of BV2 cell lines and PM, RNA-seq experiments were performed. Next, we performed principal component analysis (PCA) using DESeq2 to examine congruency among biological replicates. PCA analysis showed a good separation and high level of consistency between biological replicates of the same population in BV2 cell lines and PM (Additional file 2: Figure S2). According to the above criteria, 237 genes for $2 \mathrm{~h}$ and 331 genes for $4 \mathrm{~h}$ were differentially regulated following LPS treatment BV2 cell lines. Of these, 205 and 299 genes were up-regulated, and 32 genes were down-regulated at 2 and $4 \mathrm{~h}$, respectively, after LPS treatment in BV2 cell lines (Fig. 2b; Additional file 3: Figure S3). Surprisingly, we found significant generational differences in PM, in which 531 genes for $2 \mathrm{~h}$ and 1286 genes for $4 \mathrm{~h}$ were differentially regulated. Of these, 362 and 946 genes were up-regulated, and 169 and 340 genes were down-regulated at 2 and $4 \mathrm{~h}$, respectively, after LPS treatment (Fig. 2c; Additional file 3: Figure S3), in contrast to our previous studies showing that differentially expressed genes in the BV2 cell lines were less pronounced at 2 and $4 \mathrm{~h}$ after LPS treatment [20,31]. In this analysis, we have increased biological duplicate to biological triplicate RNA-seq as well as $P$ value less than 0.01 for differential expression genes, which reduces the number of differential expression genes in the BV2 cell lines after 2- and 4-h LPS treatment. In addition, differences in the passage number and intrinsic variability of cells may lead to these discrepancies. The genes were grouped into several categories based on their biological processes and molecular gene ontology functions, and heat maps were generated to aid the visualization of the gene expression pattern. The top 150 inflammatory genes that were upregulated at 2 and $4 \mathrm{~h}$ after LPS stimulation in BV2 and PM cells are shown in Fig. 2a. Next, we performed functional classification analyses of the up-regulated genes using DAVID Bioinformatics Resources [27] by classifying the results into gene ontology (GO) categories (FDR 0.05) using the biological process (BP) and molecular function (MF) categories. We observed that in both BV2 cell lines and PM, the top 150 genes that were up-regulated in response to 4-h LPS stimulation were mainly involved in the immune system process and multi-organism processes (Fig. 2e, f). Because the down-regulated genes were not associated with inflammation, only the up-regulated genes were further studied. We confirmed using GO analysis (FDR 0.05) and DAVID Bioinformatics Resources that the top 150 transcripts that were down-regulated by 4-h LPS stimulation in PM were associated with the developmental process and regulation of GTPase activity (Additional file 4: Figure S4).

\section{Common and unique characteristics of PM versus BV2 cell lines}

To further investigate common and unique characteristics between LPS-treated PM or the BV2 cell lines, we again used RNA-seq data to compare the transcriptome of BV2 cell lines with that of PM. In a similar approach (see the "Methods" section), we compared the transcripts in LPS-treated BV2 cell lines with those of PM. Differential expression analysis clearly revealed that LPS elicited the induction of a unique gene set in response to stimulation with this TLR ligand at the 2- and 4-h time point in BV2 cell lines and PM cells (Fig. 2d; Additional file 3: Figure S3) suggesting a substantial number of dissimilarities between the two cell types. PM cells upregulated 220 for $2 \mathrm{~h}$ and 682 genes for $4 \mathrm{~h}$ that are not common to the BV2 cell lines. In contrast, BV2 cell lines up-regulated 63 for $2 \mathrm{~h}$ and 35 genes for $4 \mathrm{~h}$ that are not common to the PM cells (Fig. 2d; Additional file 3: Figure S3). The unique gene set is presented at the 2and 4-h time point in BV2 cell lines in Additional file 5: Table S1. However, PM and the BV2 cell lines also had similarities in their transcriptomes. Of the up-regulated genes, BV2 cell lines and PM shared 142 genes for $2 \mathrm{~h}$ and 264 genes for $4 \mathrm{~h}$ following LPS treatment (Fig. 2d; Additional file 3: Figure S3). Importantly, this technology allowed us to identify several specific gene families involved in immune responses that were uniquely altered in LPS-treated PM cells. We found that LPS elicited the induction of unique 10 cytokines, 9 chemokines, 13 (interferon (IFN))-regulated genes (IRGs), 9 TFs, 3 epigenetic regulators, and 11 undetected transcripts in response to stimulation with this TLR ligand at the 4-h time point in PM cells (Figs. 4b, 5b, and 6f). The following inflammatory response- and immune response-related genes were markedly affected only in PM: cytokines/chemokines (CCL6, CCL8, CX3CL1, CXCL1, CXCL3, CXCL9, CXCL11, CXCL16, IL12B, IL18BOS, IL18BP, IL19, IL23A, IL27, IRAK1BP1, SOCS1, TNFSF11A, and TNFSF15), IRGs (GBP2B, GBP4, GBP9, GBP10, GBP11, IFI44I, IFIH1, IFNB1, etc.), TFs (IRF2, IRF5, IRF8, STAT5A, etc.), epigenetic regulators (KDM1A, NSD3, and SETDB2), and undetected transcripts (CLEC4A1, CLEC7, CLEC7A, GPR18, MMP3, MMP9, MMP12, etc.). These data suggest that following LPS treatment, PM express a unique set of genes, distinct from that of 


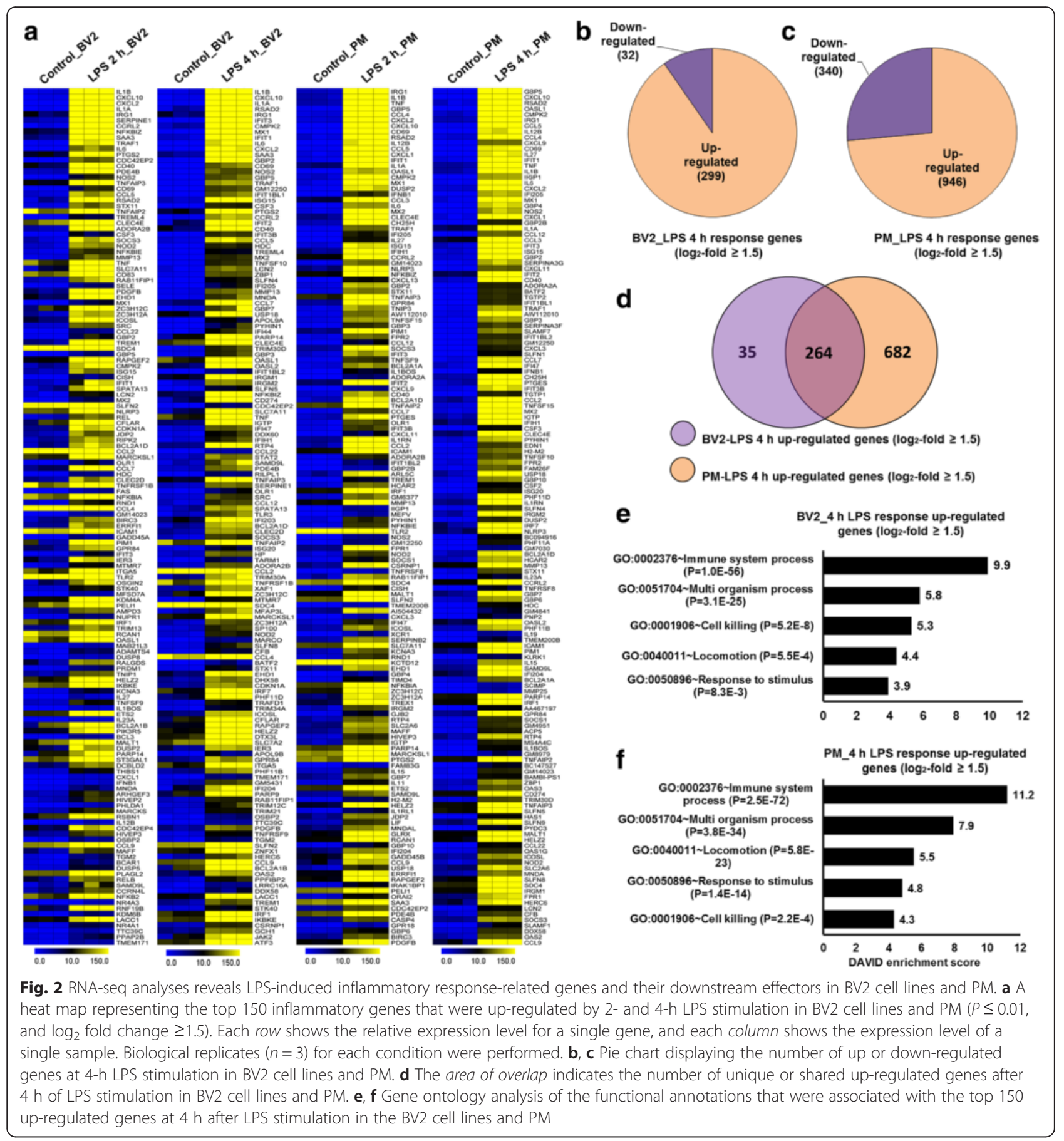

BV2 cell lines, which may offer potential targets for further investigations into microglia biology.

\section{Canonical pathway prediction modulated through TLR4-stimulated PM and BV2 cell lines}

To gain further understanding into the molecular functions of up-regulated genes, we performed IPA (IPA, Ingenuity Systems, http://www.ingenuity.com) [28] to identify the canonical pathways that represent the relevant molecular functions based on functional knowledge inputs. IPA analysis of transcriptome profiling data revealed the highly statistically significant regulation of well-known TLR4mediated pathways that are important in immune responses, including genes involved in the roles of pattern recognition receptors during the recognition of bacteria and viruses, interferons, and NF-kB signaling, in BV2 cell lines and PM (Fig. 3a, b). Other notable pathways included the TREM1, toll-like receptor, and death receptor signaling 


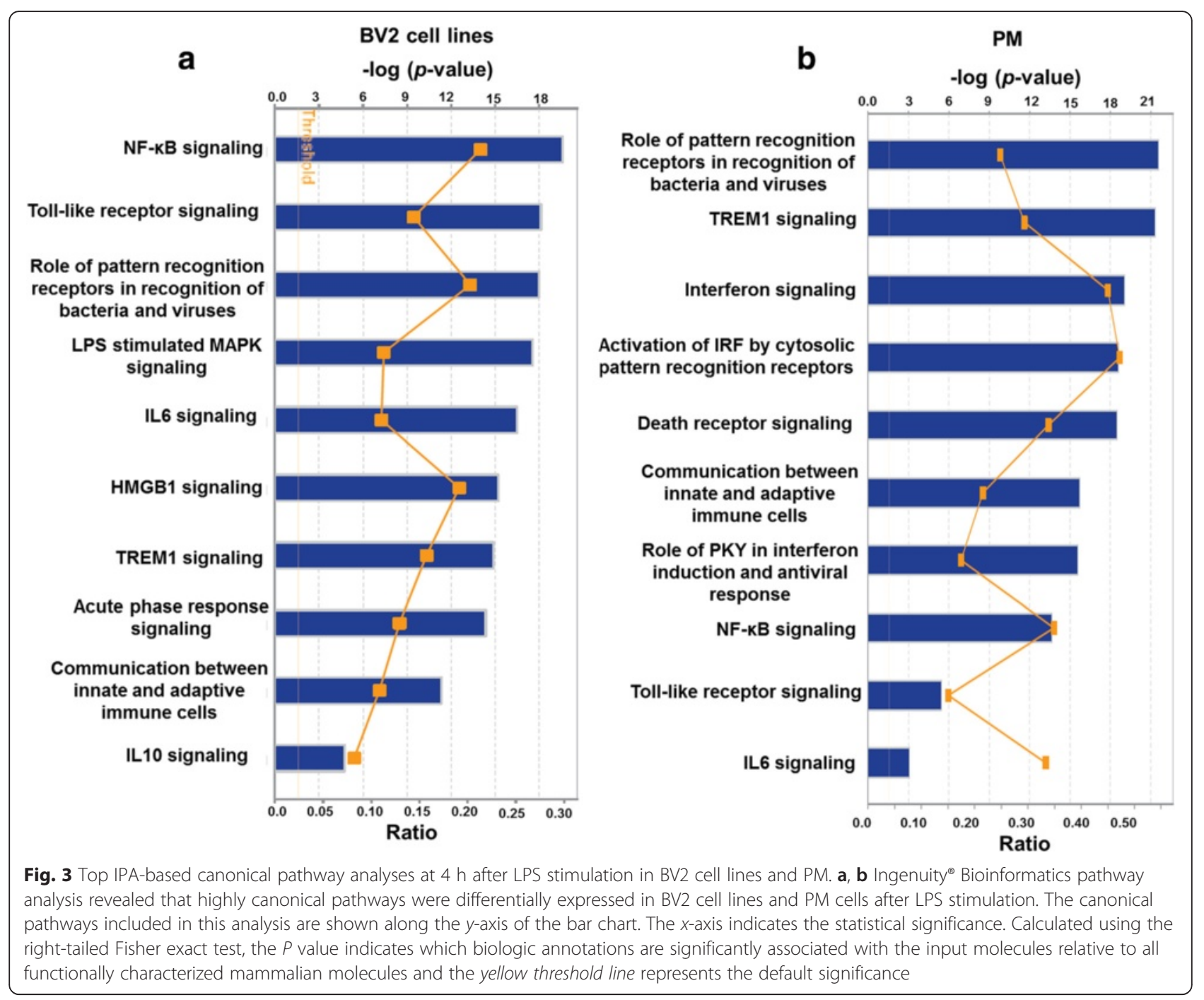

pathways. The up-regulation of these functions in response to stimulation with TLR4 stimulation is interesting and indicates that our approach for comparing BV2 cell lines and PM is strong but rather predictable. Interestingly, the pathways that scored as unique in PM relative to microglial cell lines were involved in interferon signaling. The biggest difference between PM and microglial cell lines was therefore a difference in interferon signaling according to IPA analysis. This may reflect the interferon signaling that is known to occur in PM following immune activation.

The expression of proinflammatory cytokines, chemokines, and interferon response transcripts are highly expressed in PM than BV2 cell lines following 2- and 4-h stimulation with TLR4

To identify differences in the regulation of these genes in PM compared to BV2 cell lines, cells were treated with LPS to analyze change in cytokine, chemokine, and interferon response gene expression. Our RNA-seq analysis revealed that almost all of the proinflammatory cytokines/chemokines were more highly induced in PM than in the BV2 cell lines (Fig. 4a, c, d). Importantly, PM expressed several unique cytokines/chemokines that were not expressed in the BV2 cell lines (Fig. 4b). These included CCL6, CCL8, CX3CL1, CXCL1, CXCL3, CXCL9, CXCL11, CXCL16, IL12B, IL18BOS, IL18BP, IL19, IL23A, IL27, IRAK1BP1, SOCS1, TNFSF11A, and TNFSF15. Interestingly, the CXCL10-associated CXCR3-binding molecules CXCL9 and CXCL11 were highly enriched only in PM cells, while CXCL4 was not. The growth-regulated oncogenes (GRO) (CXCL1 and CXCL3) also displayed a similar trend: they were expressed only in PM. Monocyte chemoattractant proteins (MCP) 1 and 3 (CCL2 and CCL7, respectively) were more highly induced in PM than in the BV2 cell lines (Fig. 4a). However, CCR7, which is a dendritic cell antigen that is expressed in microglia in inflamed central nervous system (CNS) tissues [32], stromal-derived factor 1 (SDF-1, also CXCL12), and the recently discovered chemokine 


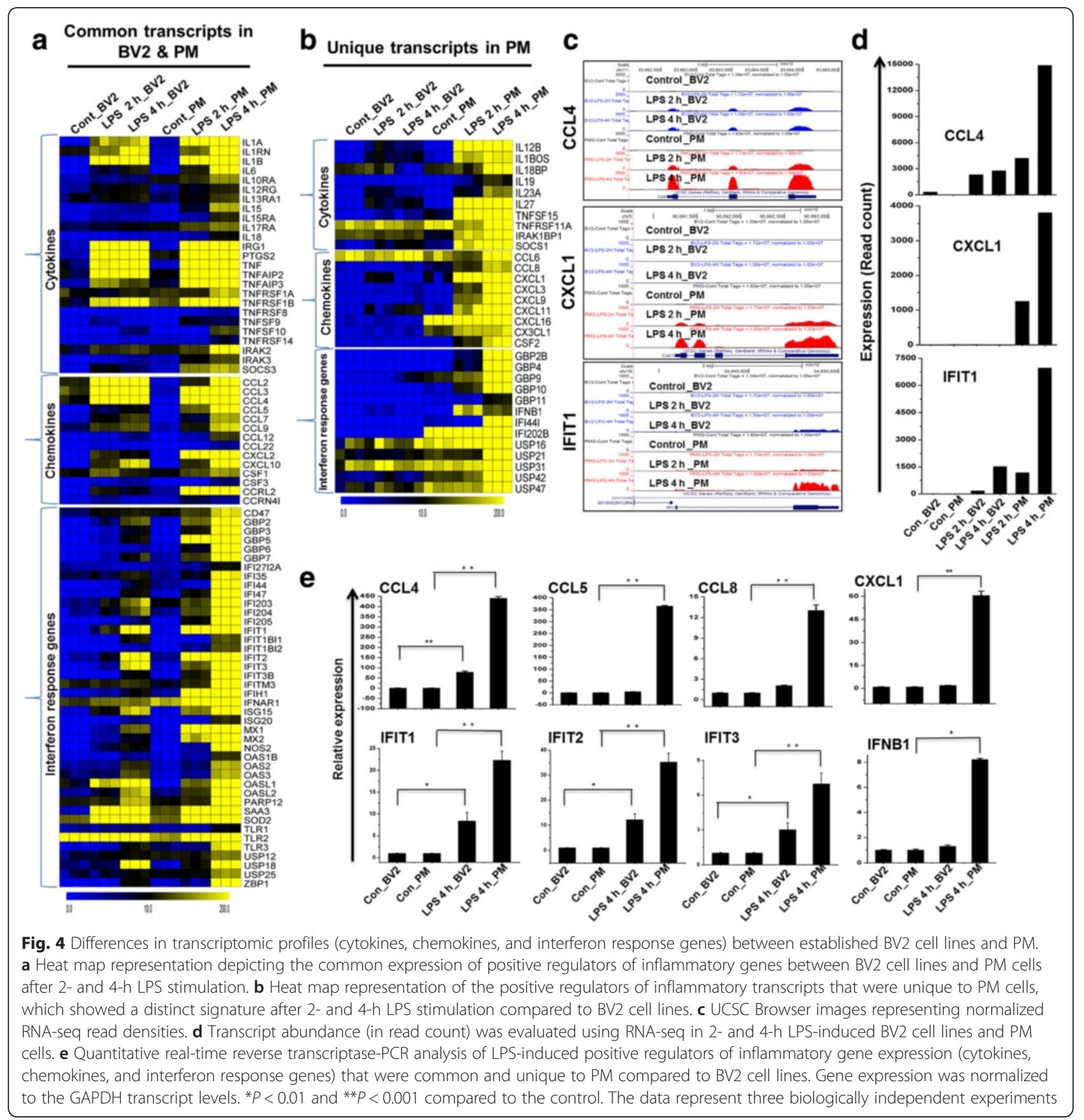

CCL25 were not induced in either PM or the BV2 cell lines. Engagement of TLR activates signaling pathways that lead to transcriptionally induce hundreds of IRGs. Type I and type II have well-described antiviral properties, and most of the IRGs are regulated by both type I and type II IFNs [33]. These IRGs included factors known to be involved in antiviral responses. To further characterize the similarities and differences between the analyzed BV2 cell lines and PM, we compared the levels of expression of IRGs. These highly induced genes included the IFN-induced protein with tetratricopeptide (IFIT) family gene IFIT1 (p56), IFIT2, IFIT3,
IFIH1, IFITM3, the IRF3-dependent gene ISG15 [34], ISG20, IFI35, IFI44, IFI47, IFI203, IFI204, IFI205, OASL, OASL2, OAS3, etc., with their expression in PM being higher than their expression in the BV2 cell lines (Fig. 4a). Importantly, the PM expressed several IRGs that were not expressed in the BV2 cell lines (Fig. 4b). RNA-seq analysis revealed that 43 IRGs were significantly up-regulated in both BV2 cell lines and PM. Importantly, we found that almost all IRG BV2 cell line response was weaker than the response of PM. A better understanding of the consequences of LPS-stimulated IRG production in PM warrants 
a comprehensive investigation. These included GBP4, GBP6, IFNB1, and MX2 [35]. However, we did not detect other IRGs, such as IFITM1 and GBP1, in either the PM or the BV2 cell lines. These data strongly suggest that LPS induces proinflammatory cytokines, chemokines, and IRGs more strongly and broadly in PM cells than in BV2 cell lines.

\section{PM are hyperresponsive and express higher levels of multiple families of TFs than BV2 cell lines following 2- and 4-h stimulation with TLR4}

To better understand the relationship between PM and BV2 cell lines, we reassessed the RNA-seq data. TFs, including IRF, Kruppel-like factor (KLF), NF-kB, and signal transducer and activator of transcription (STAT), are important in inflammatory diseases [36, 37]. The following TF families exhibited the most dramatic levels of induction following LPS challenge in PM: the NF-kB group of TFs (NF-kBIA, NF-kBIB, NF-kBID, NF-kBIE, NF-kBIZ, NF-kB1, NF-kB2, REL, and RELB), the interferon group of TFs (IRF1, IRF2, IRF5, IRF7, IRF8, and IRF9), and the STAT group of TFs (STAT1, STAT2, STAT3, and STAT5A) (Fig. 5a-d). However, other members of the IRF and STAT families were unaffected by treatment with LPS, suggesting that LPS induces TFs in a highly selective manner in microglia. More importantly, in agreement with a previous study performed in our laboratory, we observed that IRF2, IRF5, STAT1, STAT2, and STAT5A were not expressed in LPS-stimulated BV2 cell lines [20], suggesting that these TFs might be important regulators of the selective inflammatory gene expression that occurs in PM. In addition, our RNA-seq analysis revealed that these TFs were more highly induced in PM than in BV2 cell lines. Furthermore, we also found other groups of TFs (ATF3, E2F5, ETS2, and FOXP4) were highly up-regulated in PM (Fig. 5a, b).

Next, we conducted a TF motif analysis to analyze LPSinduced gene expression in BV2 cell lines and PM. We used the Pscan software tool [29] to perform an in silico computational analysis to determine the over-represented cis-regulatory elements within the 5 '-promoter regions of coordinately regulated genes. Applying this score to the promoters of the genes that were differentially expressed in response to LPS revealed that the putative binding sites for eight TFs (IRF1, IRF2, STAT1, STAT2, NF-kB1, RELA, SP1, and SPI1) were significantly enriched in PM (Fig. 5f), suggesting that these TFs might be involved in the regulation of LPS-induced gene expression in PM. Nevertheless, further TF-targeted studies are required to validate this regulation in PM. In addition to a TF motif analysis, we also used IPA software [28] to identify the target genes that were directly or indirectly activated by the TFs that were identified to be activated in response to LPS stimulation. Importantly, we found that the expression of the majority of the cytokines and chemokines was directly regulated by the TFs that were identified as selectively enriched in PM, including IRF1, IRF2, STAT1, STAT2 (Fig. 5g and Table 2). To further functionally classify the STAT1, and IRF1-regulated genes, they were functionally annotated using the DAVID 6.8 software package [27]. Interestingly, we observed strong enrichment in the GO terms for the IRF1, and STAT1 regulated transcripts that were associated with immune system processes in the LPS-stimulated PM (Fig. 5h), suggesting that IRF1, IRF2, STAT1, and STAT2 might also be involved in the regulation of inflammation in microglia.

\section{A comparison of epigenetic regulators and undetected transcripts between BV2 cell lines and PM following 2- and 4-h stimulation with TLR4}

To further characterize the degree of similarity or dissimilarity between BV2 cell lines and PM, we compared the most significant epigenetic regulators between these two groups. We defined this as genetic control via factors other than the DNA sequence [38]. A high level of induction of the epigenetic regulator histone methyltransferase (SETDB2) has recently been demonstrated to potentiate innate immune responses [39]. We previously showed that DNA methyltransferase (DNMT3L) and histone demethylases (KDM4A) were specifically upregulated after LPS stimulation in microglial BV2 cell lines [20]. To our surprise, in this study, we found that in addition to DNMT3L and KDM4A, histone-lysine $N$ methyltransferase NSD3, lysine (K)-specific demethylase 1a (KDM1A), and histone methyltransferase SETDB2 were also significantly up-regulated in PM compared to BV2 cell lines, indicating that KDM1A, NSD3, and SETDB2 may be involved in the selective regulation of PM functions (Fig. 6a-c). However, we could not identify any histone deacetylase genes (HDAC) in LPS-induced BV2 cell lines and PM (Fig. 6a). The neuroinflammatory response to activation states has also been implicated to involve microRNAs (miRNAs). miRNAs are a class of small non-coding RNA molecules, $\sim 22$ nucleotides in length that function in the post-transcriptional regulation of gene expression. Dysregulation of miRNA expression has been demonstrated to play critical roles in the pathogenesis of CNS diseases. For example, during the onset of experimental autoimmune encephalomyelitis (EAE), miR124 is down-regulated in microglia, while transfection of miR-124 leads to marked reduction in the severity of EAE, suggesting that miR-124 can be a target for neuroprotection [40]. Nevertheless, in the presence of LPS exposure, detailed miRNA profiling will be required to determine the unique miRNA signature and their selective roles to regulate inflammatory genes in microglial BV2 cell lines. This is an exciting area that we are keenly pursuing further. In addition to differentially expressed cytokines/ 


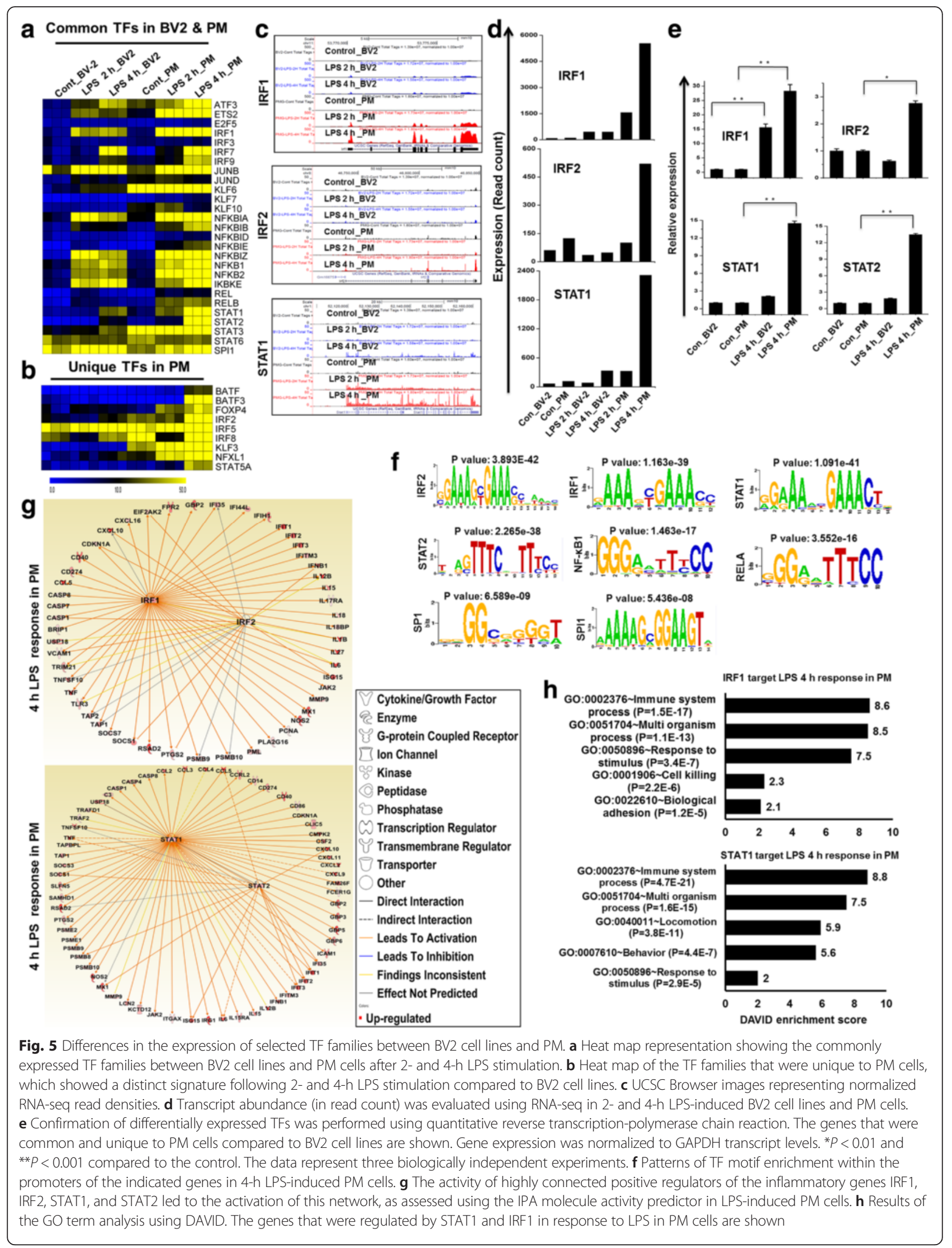


Table $\mathbf{2}$ Leads to activation of inflammatory genes by identified TFs in response to 4-h LPS stimulation in PM

\begin{tabular}{llll}
\hline $\begin{array}{l}\text { STAT1 predicted to be activated } \\
\text { (65 genes) }(P=1.41 \text { E-74) }\end{array}$ & $\begin{array}{l}\text { STAT2 predicted to be activated } \\
(14 \text { genes })(P=1.03 \mathrm{E}-41)\end{array}$ & $\begin{array}{l}\text { IRF1 predicted to be activated } \\
\text { (51 genes) }(P=4.98 \mathrm{E}-61)\end{array}$ & $\begin{array}{l}\text { IRF2 predicted to be activated } \\
(21 \text { genes })(P=3.55 \mathrm{E}-35)\end{array}$ \\
\hline CASP1, CASP4, CASP8, CCL2, CCL3, & CCL5, CXCL10, IFI35, IFIT1, IFIT2, & TRIM21, TNFSF10, TNF, TLR3, TAP2, & USP18, VCAM1, TRIM21, TNFSF10, \\
CCL4, CCL5, CCRL2, CD14, CD274, & IFIT3, IL6, ISG15, MX1, PSMB8, & TAP1, SOCS7, SOCS1, RSAD2, PTGS2, & TLR3, TAP2, TAP1, SOCS1, PTGS2, \\
CD40, CD86, CDKN1A, CLIC5, CMPK2, & RSAD2, SOCS1, TNF, TNFSF10 & PSMB9, PSMB10, PML, PLA2G16, & PSMB9, PSMB10, ISG15, IL6, IL1B, \\
CSF2, CXCL10, CXCL11, CXCL2, & & PCNA, MX1, NOS2, MMP9, JAK2, & IL12B, IFNB1, IFI35, EIF2AK2, \\
CXCL9, FAM26F, FCER1G, GBP2, & & ISG15, IL6, IL27, IL1B, IL18BP, IL18, & CXCL10, CDKN1A, CASP1 \\
GBP3, GBP5, GBP6, ICAM1, IFI35, & IL17RA, IL15, IL12B, IFNB1, IFITM3, \\
IFIT1, IFIT2, IFIT3, IFITM3, IFNB1, IL12B, & IFIT3, IFIT2, IFIT1, IFIH1, IFI44L, IFI35, \\
IL15, IL15RA, IL6, IRG1, ISG15, ITGAX, & GBP2, FPR2, EIF2AK2, CXCL16, \\
JAK2, KCTD12, LCN2, MMP9, MX1, & CXCL10, CDKN1A, CD40, CD274, \\
NOS2, PSMB10, PSMB9, PSMB8, & CCL5, CASP8, CASP7, CASP1, BRIP1, \\
PSME1, PSME2, PTGS2, RSAD2, & USP18, VCAM1 \\
SAMHD1, SLFN5, SOCS1, SOCS3, & & \\
TAP1, TAPBPL, TNF, TNFSF10, TRAF2, & & \\
TRAFD1, USP18, C3 & & \\
\hline
\end{tabular}

chemokines, TFs, and epigenetic regulators, annotation of the RNA-seq data also revealed approximately 11 previously undetected genes that were specifically up-regulated in PM. Furthermore, we found that approximately 40 previously undetected genes were significantly up-regulated in PM compared to BV2 cell lines (Fig. 6e-g).

\section{Confirmation of differentially expressed genes by qRT-PCR}

A large number of genes that were identified to be differentially regulated using RNA-seq analysis were subjected to validation using qRT-PCR. GAPDH was used as the reference gene. Most of the genes were selected for validation based on whether they were selectively altered by LPS stimulation in PM. To measure gene expression, mRNA was reverse transcribed into cDNA using PrimeScript TM Reverse Transcriptase (Takara Bio Inc., Shiga, Japan), and the qRT-PCR assays were repeated several times using at least three mRNA preparations from independent experiments. The results are expressed as the fold change relative to the control levels. Sixteen genes were selected for verification, and the RNA-seq expression patterns were confirmed for 14 of these genes (CCL4, CCL5, CCL8, CXCL1, IFIT1, IFIT2, IFIT3, IFNB1, IRF1, IRF2, STAT1, STAT2, KDM4A, and SETDB2; Figs. 4e, 5e, and 6d). Two genes (KLF7 and IRAK3) were found to be nonsignificantly altered (data not shown) in the qRT-PCR analysis compared to the RNA-seq experiments. Furthermore, we previously confirmed that the levels of the cytokines, chemokines, and several transcripts in the supernatants were significantly up-regulated in PM cells compared to the BV2 cell lines [31].

\section{Discussion}

Using an RNA-seq approach, in the present study, we show that unique transcriptional changes occur in mouse PM following inflammatory stimulation that distinguished these cells from microglial BV2 cell lines. Although a few studies have attempted to use microarrays to produce comparative transcriptional profiles between BV2 cell lines and PM, such studies are limited because this technology provides only a semi-quantitative assessment of the transcriptome $[10,16]$. The strength of our analysis, which was aimed at obtaining comprehensive and comparative transcriptional profiles of responses to inflammatory stimulation, was enhanced by the use of RNA-seq to analyze differences between BV2 cell lines and PM. The data obtained in our study correlate to a large extent with those demonstrated by other studies, including $[10,16]$. In contrast to our findings, the above studies demonstrated that in the presence of LPS (100 ng/ $\mathrm{ml})$, PM cells up-regulated only 118 genes at the 4-h time point and of the up-regulated genes, BV2 cell lines shared most of the genes to PM using microarray experiments $[10,16]$. The present study not only greatly extends earlier findings but also distinguishes between BV2 cell lines and PM in response to LPS. These results allowed us to discover novel transcriptional alterations that occurred in PM but were not detected in BV2 cell lines. The surprising and unexpected finding of our study was that PM reacted stronger to LPS and that therefore, a much larger number of transcripts, including many novel transcripts, were altered in PM than in BV2 cell lines. Therefore, there is a potentially enormous qualitative difference in responses to inflammatory stimulus between PM and BV2 cell lines. Importantly, Cao et al. [41] reported that upon secondhit LPS exposure, heme oxygenase 1 (HMOX1) and fructose-1,6-bisphosphatase (FBP) genes as well as HDAC 1, 2, 4, and 6 were uniquely differentially expressed in microglia, which have potential role for desensitization. In our RNA-seq data, we could not identify any histone deacetylases as well as FBP genes in LPS-induced BV2 cell lines and PM. However, we observed HMOX1 was significantly expressed in both BV2 cell lines and PM in response to 4-h LPS (Additional file 6: Figure S5). Nevertheless, whether this gene has any functional role on LPS-mediated modulation of microglia activation will require further study. 


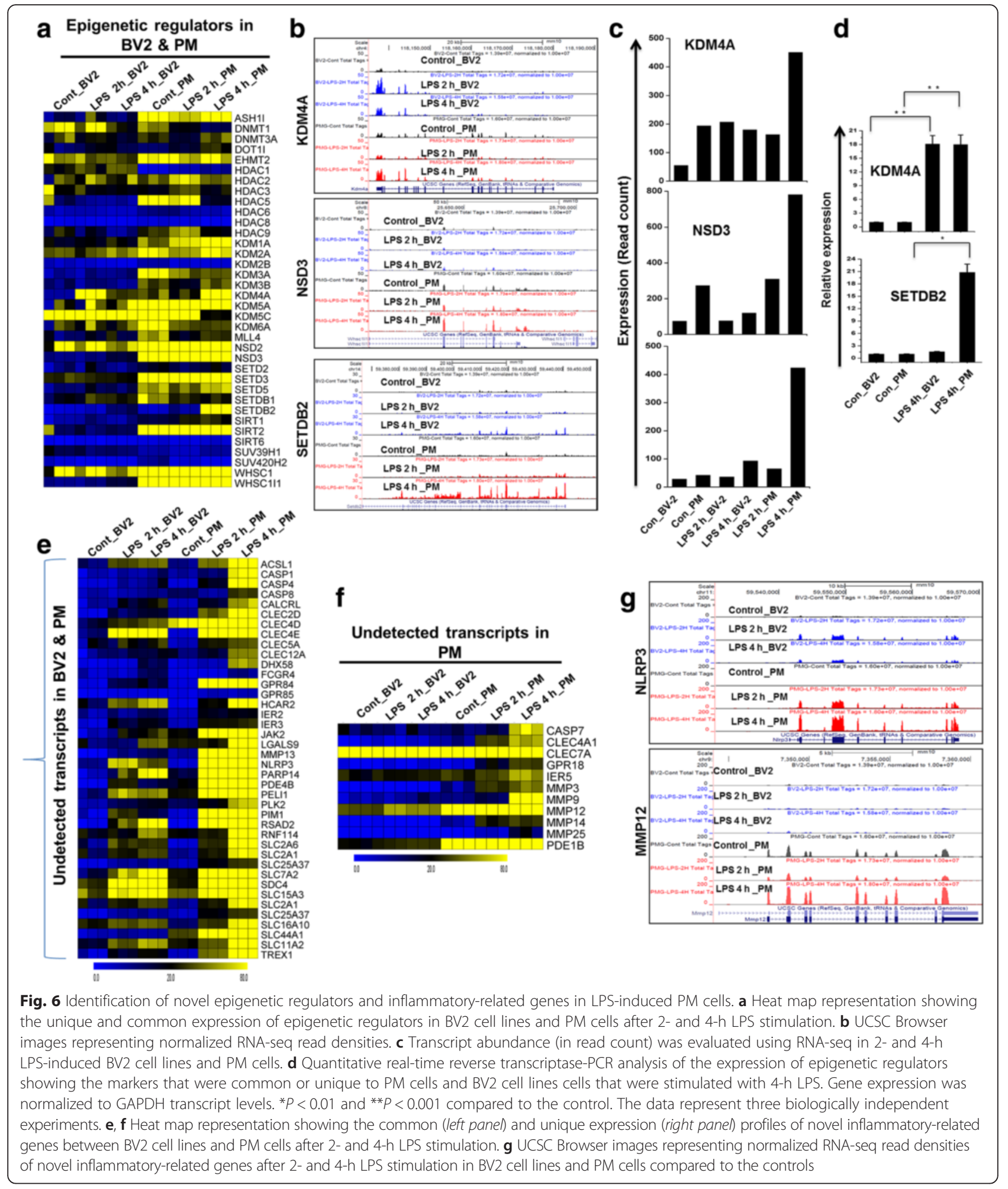

These results showed that cytokines/chemokines, antiviral genes, and IRGs that are associated with inflammation were significantly up-regulated in response to LPS and that these changes were stronger in PM than in BV2 cell lines microglia (Fig. 4a-e). Both the number of genes and the extent of the fold changes in the commonly altered genes were significantly more modulated in PM compared to BV2 cell line microglia. PM cells upregulated 220 for $2 \mathrm{~h}$ and 682 genes for $4 \mathrm{~h}$ that are not common to the BV2 cell lines (Fig. 2d; Additional file 3: 
Figure S3). Importantly, our RNA-seq analysis is the first to identify several important differences in the expression patterns of cytokines/chemokines, antiviral genes, and IRGs that were not previously identified to be activated by LPS in BV2 cell lines but that were found to be altered in PM (Fig. 4b). In particular, this technology allowed us to identify 10 cytokines, 9 chemokines, and 13 IRG genes that were uniquely altered in LPS-stimulated PM cells. The following inflammatory response- and immune response-related genes were markedly affected only in PM: cytokines/chemokines (CCL6, CCL8, CX3CL1, CXCL1, CXCL3, CXCL9, CXCL11, CXCL16, IL12B, IL18BOS, IL18BP, IL19, IL23A, IL27 IRAK1BP1, SOCS1, TNFSF11A, and TNFSF15) and IRGs (GBP2B, GBP4, GBP9, GBP10, GBP11, IFI44I, IFIH1, IFNB1, etc.).

Cytokines and chemokines are involved in the regulation of inflammation, and the excessive production of these molecules has been associated with disease progression, severe neuroinflammation pathologies, and synaptic transmission [42]. IL12 plays an important role in early inflammatory responses to infection, and increased IL12 expression can be dangerous to the host because it is involved in the pathogenesis of a number of autoimmune inflammatory diseases, including multiple sclerosis (MS) [43]. TNFSF15 is one of the more recently identified TNF ligands. TNFSF15 is a multifunctional, specialized cytokine that is involved in the modulation of inflammation [44]. The chemokine CCL5 has been implicated in a wide array of pathological conditions in the brain and in neurodegenerative diseases. In particular, abnormal CCL5 expression was observed in the cerebrospinal fluid (CSF) of patients suffering from MS and in the CNS in mice with EAE [45]. In addition, $\mathrm{Si}$ et al. [46] reported elevated levels of CCL5 in HIV-1 viral protein R-induced human microglial cells. Importantly, BV2 cell lines exposed to these factors did not or less induce the expression of these inflammatory response-related genes than PM.

We observed that LPS significantly induced the expression of several genes known to be involved in antiviral immunity, and we found that these changes in signaling were more potent in PM than in BV2 cell lines (Fig. 4a, b). Unexpectedly, we were unable to identify IRF3 in either PM or BV2 cell lines. Although the IRF3 target gene IFNB was induced in LPS-induced PM cells, it was not induced in BV2 cell lines. Importantly, another IRF3 target gene, CXCL10, was induced in both PM and BV2 cell lines. Our results are similar to those described in a previously published report that showed the induction of IRF3 in dendritic cells and macrophages [47]. Because no IRF3 activation was observed in either type of microglia, the mechanism by which the production of CXCL10 is induced remains obscure. Interestingly, it has been demonstrated that JAK/STAT pathway is a critical player in the regulation of CXCL10 expression in virus- and cytokine-stimulated astrocytes [48]. In addition, other reports also showed that MAP kinase cascades prominently regulate CXCL10 gene expression in microglial cells [49]. Therefore, it seems likely that the LPS-induced induction of CXCL10 transcription depends on JAK/STAT or MAP kinase pathways rather than IRF3 transcriptional pathways in microglial cells. This is an exciting area that we are keenly pursuing further. Importantly, we identified IRF2 and IRF5 as significantly up-regulated only in PM (Fig. 5b, c). In addition, we observed that IRF1, IRF7, and IRF9 were more highly up-regulated in PM than in BV2 cell lines (Fig. 5a, c). Previously, it was demonstrated that IRF, IRF5, and IRF7 may be master regulators that contribute to IRGs $[37,50,51]$. Thus, it would be interesting to explore whether IRG-inducting mechanisms that do not rely on IRF3 exist in cells other than microglia or macrophages. It is therefore likely that IRF1, IRF2, IRF5, IRF7, and IRF9 do not substitute for IRF3 in PM in response to LPS stimulation. A detailed TF target analysis will be required to determine the mechanism by which IRGs are induced in PM cells.

Using this array, we also identified several TFs, including NF-kB, STAT, KLF3, BATF, JUNB, and NFXL1, that have roles in microglial activation that are not welldescribed (Fig. 5a, b). More importantly, we identified several TFs, including KLF3, BATF, BATF3, FOXP4, NFXL1, and STAT5A, that were uniquely altered in PM (Fig. 5b), suggesting that these TFs might be important regulators in the selective inflammatory gene expression that occurs in these cells. Additionally, our RNA-seq analysis revealed that these and other TFs were more highly expressed in PM than in BV2 cell lines. STAT proteins are critical mediators of immunity to pathogens that are involved in inflammatory diseases [36]. STAT1 and STAT2 are important regulatory factors in the IFN signaling pathways, and they are also essential components of cellular antiviral responses. However, STAT6, KLF9, KLF10, KLF16, and IRF2BP1 were unaffected, suggesting that the induction of TFs in microglial cells is highly selective. To further identify the conserved TFbinding motifs, we performed TF motif analysis using PM cells. Between the two ranges that were available in Pscan that were closest to our region of interest (-950 to 50 and -1000 to 0 ), the -950 - to +50 -bp range was selected for the analyses. We found that the promoters of differentially expressed genes were enriched not only for NF-kB transcription factors but also for IRF1, IRF2, STAT1, STAT2, SP1, and SPI1, as shown in Fig. $5 f$. These analyses provide the first insights into the TFbinding motifs that may be involved in regulating subsets of specific genes in response to LPS stimulation in PM. Next, we used IPA software to identify the target genes that were directly or indirectly activated by the 
identified TFs (i.e., IRF1, IRF2, STAT1, and STAT2) in response to 4-h LPS. Importantly, we found that the expression of the majority of the LPS-induced stimulated cytokines/chemokines was directly regulated by the identified TFs (Fig. $5 \mathrm{~g}$ and Table 2).

Unexpectedly, the results in our dataset show that primary microglia express high levels of several epigenetic regulators. Epigenetic regulation is likely to be one of the major mechanisms used by cells to regulate gene expression in response to environmental stimuli [38]. Recently, we showed that the histone demethylase KDM4A and the DNA methyltransferase DNMT3L were strikingly differentially expressed in LPS-induced BV2 cell lines [20]. Importantly, our RNA-seq data revealed that not only KDM4A and DNMT3L but also NSD3, KDM1A, and the SETDB2 were strikingly differentially expressed in LPSinduced PM (Fig. 6a-d). Recently, Schliehe et al. [39] demonstrated that SETDB2 was induced during infection with influenza virus and that it potentially regulates proinflammatory gene expression in macrophages. The mechanism by which NSD3 and SETDB2 become activated following LPS stimulation remains unknown. Determining how these epigenetic regulators, in combination with modified TFs, can regulate distinct sets of inflammatory genes in microglial cells would be intriguing. We suggest that the role of these epigenetic regulators in neuroinflammatory diseases should now be assessed in animal models involving TLRspecific gene deletion or overexpression. However, other histone methyltransferases and $N$-methyltransferases were not expressed in LPS-induced BV2 cell lines and PM cells.

Another interesting finding is that our RNA-seq analysis identified several important differences in the patterns of genes that were uniquely induced by LPS in PM cells (and not induced by LPS in BV2 cell lines). In particular, this technology allowed us to identify over 40 directly LPS-induced genes in these cells (Fig. 6e-g). The results show that almost all of the genes that were associated with inflammation were significantly up-regulated in response to LPS and that their up-regulation was more potent in PM than in BV2 cell lines. Both the extent of the fold changes and the 40 of genes that were changed were significantly increased in PM compared to BV2 cell lines after 2- and 4-h LPS stimulation. The directly LPS-induced genes that are known to be important during activity-regulated processes in PM include LRR and PYD domain-containing protein 3 (NLRP3), which is involved in inflammasomes in MS [52]; caspase (CASP)1; CASP4, which plays a critical role in the processing and secretion of proinflammatory molecules [53]; and PELI1, which is highly expressed in microglia and plays a key role in microglial activation during EAE induction [54]. In this study, we examined BV2 cell lines and PM cells isolated from 3-day-old ICR mice as a model of inflammation studies. Previously, other reports demonstrated that rodent microglia appear to assume similar activation states to human microglia $[55,56]$. However the chemotaxis, phagocytosis, transcription regulation, and immunological characteristics have been shown differential expression in adult versus neonatal microglia $[14,57]$. Supporting this, previous studies have also demonstrated that microglia derived from neonatal, young, and aged animals are likely to have a unique molecular expression pattern in their responses to activation due to developmental state as well as cell senescence that hinders the cell's ability to sense changes in their environment [58]. Nevertheless, in the presence of activation states, further studies are warranted to determine the unique transcriptomic signature in different microglial phenotypes as well as the mechanisms by which these LPS-induced genes are expressed and their roles in neuroinflammatory disorders involving microglia.

Microglial cell activation in neuroinflammation is thought to be critically complex. It has been generally thought that microglia can broadly exist in two different states, referred to as the "classically activated," proinflammatory M1 phenotype and "alternatively activated," reparative M2 phenotypes [59]. M1 activity can be evoked by LPS, TNF- $\alpha$, and IFN- $\gamma$; induces the production of proinflammatory cytokines, oxidative metabolites, and proteases; and is expected to act as neurotoxic cells, while M2 activation is induced by the stimulation of IL4, IL10, IL13, and TGF- $\beta$ and may be involved in wound repair and remodeling as well as the production of antiinflammatory cytokines [60, 61]. Previous studies have shown that adding IL4 before LPS or simultaneous addition of LPS and IL4 significantly dampened the proinflammatory cytokines compared with LPS alone in rat PM cells $[62,63]$. These reports are supported by recent results showing that IFN- $\gamma$ - plus TNF- $\alpha$-exposed rat PM cells had significantly increased inflammatory mediators as well as receptors/enzymes related to phagocytosis and ROS production but not by IL4 [64]. Similarly, other laboratories provide evidence that in the presence of IFN- $\gamma$, but not to IL4 or IL10, BV2 cell lines had significantly increased TNF- $\alpha$, iNOS, and NO levels and drastically increased expression of NADPH oxidase (NOX2), which plays a critical role in traumatic brain injury (TBI) [65]. Interestingly, Ghosh et al. recently demonstrated that combination of cyclic adenosine monophosphate (cyclic AMP) and IL4, but neither alone, showed ameliorated production of proinflammatory cytokines (TNF- $\alpha$ and IP-10) as well as ROS production in the BV2 cell lines and PM cells [66]. Moreover, there have been data indicating that $\beta$-amyloid $(A \beta)$ induces the expression of proinflammatory cytokines in microglia cells, which could lead to AD $[67,68]$, and IL4 treatment of rat PM cells enhanced uptake and degradation of $A \beta$ [69]. 
Nevertheless, under classically or alternatively activated conditions, further studies are warranted to determine the unique transcriptomic signature between BV2 cell lines and PM cells.

Overall, our RNA-seq data provide novel insight into the transcriptional differences between cell lines and PM. It is abundantly clear that commonly used microglial BV2 cell lines do not express the same molecular signature as PM after LPS stimulation. The identification of a definitive quantitative PM transcriptome may be useful in determining the features that account for microglia neuroinflammatory functions and their neurotoxic versus neuroprotective properties. In the future, this model can be extended to include data from other high-dimensional surveys, such as microRNA, ChIP-seq, and proteomics, to provide further insight into the global gene regulation processes that occur in LPS-induced microglia.

\section{Conclusions}

In summary, using RNA-seq, we compared the transcriptome changes of microglia BV2 cell lines and PM following stimulation of LPS. Our study demonstrates that PM reacted stronger to LPS and that therefore, a much larger number of transcripts, including different immunoregulatory (cytokines, chemokines, IRGs, etc.) genes, different TFs, and epigenetic regulators, as well as undetected transcripts, were altered in PM than in BV2 cell lines. Furthermore, we identified several specific gene families involved in immune responses that were uniquely altered in LPS-treated PM cells. Our findings thus provide new insights into microglial biology and probably will require more focus on PM activation studies.

\section{Additional files}

Additional file 1: Figure S1. Morphology and quantification of CD11bpositive microglial cells. (A) Photomicrographs are representative images of BV2 cell lines and PM at $4 \mathrm{~h}$ with and without (control) treatment with LPS. Images are at $\times 20$ magnification. (B) Microglial purity is accomplished using flow cytometry. 95.83 and $92.32 \%$ of cells obtained were BV2 cell lines and PM microglia, respectively, as quantified by CD11b. The labeled cells are represented by the purple- and red-shaded populations. (TIF $21196 \mathrm{~kb}$ )

Additional file 2: Figure S2. PCA analysis of LPS-treated and untreated BV2 cell lines and PM cells. The first principal component (PC1) separates the untreated and LPS-treated samples, while the second principal component (PC2) separates the biological replicates of the same population in BV2 cell lines and PM. (TIF $9666 \mathrm{~kb}$ )

Additional file 3: Figure S3. Identification of unique and shared genes after 2-h LPS stimulation in BV2 cell lines and PM. Pie chart displaying the number of up or down-regulated genes at 2-h LPS stimulation in BV2 cell lines and PM (upper panel). The area of overlap indicates the number of unique or shared up-regulated genes after $2 \mathrm{~h}$ of LPS stimulation in BV2 cell lines and PM (lower panel). (TIF $15155 \mathrm{~kb}$ )

Additional file 4: Figure S4. Functional annotations of 4-h LPSinducible down-regulated genes in PM. Gene ontology analysis of functional annotations (biological processes) associated with the top 150 LPS-inducible down-regulated genes at $4 \mathrm{~h}$ in PM compared to the control. (TIF $5365 \mathrm{~kb}$ )
Additional file 5: Table S1. Unique up-regulated genes after 2 and $4 \mathrm{~h}$ of LPS stimulation in BV2 cell lines. (DOC 98304 kb)

Additional file 6: Figure S5. UCSC Browser images (left panel) representing normalized RNA-seq read densities and transcript abundance (right panel) (in read count) was evaluated in HMOX1 gene using RNA-seq in 2- and 4-h LPS-induced BV2 cell lines and PM cells. (TIF 16998 kb)

\section{Abbreviations}

$A \beta, \beta$-amyloid; BP, biological process; CASP, caspase; CNS, central nervous system; CSF, cerebrospinal fluid; cyclic AMP, cyclic adenosine monophosphate; DAVID, Database for Annotation, Visualization and Integrated Discovery; DEG, differentially expressed gene; DMEM, Dulbecco's modified Eagle's medium; EAE, experimental autoimmune encephalomyelitis; FBP, fructose-1,6-bisphosphatase: FBS, fetal bovine serum; GAPDH, glyceraldehyde-3-phosphate dehydrogenase: $\mathrm{GO}$, gene ontology; GRO, growth-regulated oncogenes; HDAC, histone deacetylase genes; HMOX, heme oxygenase; IACUC, Institutional Animal Care and Use Committee; IFIT, IFN-induced protein with tetratricopeptide; IFN, interferon; IL, interleukin; IPA, Ingenuity Pathway Analysis; IRG, IFNregulated gene; KDM, lysine (K)-specific demethylase; KLF, Kruppel-like factor; LPS, lipopolysaccharide; MCP, monocyte chemoattractant proteins; MCSF, macrophage colony-stimulating factor; MF, molecular function; miRNA, microRNA; MS, multiple sclerosis; NLRP, LRR and PYD domain-containing protein; NO, nitric oxide; NOX, NADPH oxidase; PCA, principal component analysis; $\mathrm{PGE}_{2}$, prostaglandin E2; PM, primary microglia; RNA-seq, RNA sequencing; ROS, reactive oxygen species; SDF, stromal-derived factor; TBI, traumatic brain injury; TF, transcription factor; TGF- $\beta$, transforming growth factor beta; TLR, toll-like receptor; TNF-a, tumor necrosis factor-alpha

\section{Acknowledgements}

Not applicable.

\section{Funding}

This work was supported by a National Research Foundation of Korea (NRF) grant that was funded by the Korean government (MSIP)

(2013R1A1A3011026 to KHJ and 2011-0030049 to YGC). The funders had no role in the study design, data collection and analysis, or preparation of the manuscript.

\section{Availability of data and materials}

The acquired data were deposited in the Gene Expression Omnibus database under dataset accession nos. GSE79898 and GSE80304.

\section{Authors' contributions}

$A D$ conceived and led the project. $A D$ wrote and drafted the manuscript. $A D, S H K, S A$, and TY performed all of the experiments and data collection. $A D$, JCC, YGC, YSL, KHJ, and KSP performed the bioinformatics and transcriptional data analyses. YGC supervised the study. All of the authors were involved in proofreading the manuscript. All of the authors read and approved the final manuscript.

\section{Competing interests}

The authors declare that they have no competing interests.

\section{Consent for publication}

Not applicable.

\section{Ethics approval and consent to participate}

All experimental protocols were performed in accordance with the Institutional Animal Care and Use Committee (IACUC) guidelines and approved by the IACUC committee of Hanyang University (HY-IACUC-20140164A and HY-IACUC-2015-0075).

\section{Author details}

'Institute of Natural Science and Technology, Hanyang University, Ansan 15588, Republic of Korea. ${ }^{2}$ Department of Molecular and Life Sciences, Hanyang University, Ansan 15588, Republic of Korea. ${ }^{3}$ Department of Bionanotechnology, Hanyang University, Seoul 04673, Republic of Korea. 


\section{Received: 11 December 2015 Accepted: 27 June 2016}

\section{Published online: 11 July 2016}

\section{References}

1. Streit WJ, Mrak RE, Griffin WS. Microglia and neuroinflammation: a pathological perspective. J Neuroinflammation. 2004;1(1):14.

2. Graeber MB, Streit WJ. Microglia: biology and pathology. Acta Neuropathol. 2010;119(1):89-105.

3. Lawson LJ, Perry VH, Dri P, Gordon S. Heterogeneity in the distribution and morphology of microglia in the normal adult mouse brain. Neuroscience. 1990;39(1):151-70

4. More SV, Kumar H, Kim IS, Koppulla S, Kim BW, Choi DK. Strategic selection of neuroinflammatory models in Parkinson's disease: evidence from experimental studies. CNS \& neurological disorders drug targets. 2013;12(5):680-97.

5. Falsig J, van Beek J, Hermann C, Leist M. Molecular basis for detection of invading pathogens in the brain. J Neurosci Res. 2008;86(7):1434-47.

6. Bodea LG, Wang Y, Linnartz-Gerlach B, Kopatz J, Sinkkonen L, Musgrove R, Kaoma T, Muller A, Vallar L, Di Monte DA et al. Neurodegeneration by activation of the microglial complement-phagosome pathway. The Journal of neuroscience : the official journal of the Society for Neuroscience. 2014;34(25):8546-56.

7. Fan K, Li D, Zhang Y, Han C, Liang J, Hou C, Xiao H, Ikenaka K, Ma J. The induction of neuronal death by up-regulated microglial cathepsin $\mathrm{H}$ in LPS-induced neuroinflammation. J Neuroinflammation. 2015;12:54

8. Bi XL, Yang JY, Dong YX, Wang JM, Cui YH, Ikeshima T, Zhao YQ, Wu CF. Resveratrol inhibits nitric oxide and TNF-alpha production by lipopolysaccharide-activated microglia. Int Immunopharmacol. 2005;5(1):185-93.

9. Lee J, Kim YS, Choi DH, Bang MS, Han TR, Joh TH, Kim SY. Transglutaminase 2 induces nuclear factor-kappaB activation via a novel pathway in BV-2 microglia. J Biol Chem. 2004;279(51):53725-35.

10. Henn A, Lund S, Hedtjarn M, Schrattenholz A, Porzgen P, Leist M. The suitability of BV2 cells as alternative model system for primary microglia cultures or for animal experiments examining brain inflammation. Altex. 2009;26(2):83-94.

11. Crotti A, Benner C, Kerman BE, Gosselin D, Lagier-Tourenne C, Zuccato C, Cattaneo E, Gage FH, Cleveland DW, Glass CK. Mutant Huntingtin promotes autonomous microglia activation via myeloid lineage-determining factors. Nat Neurosci. 2014;17(4):513-21.

12. Lund $S$, Porzgen $P$, Mortensen AL, Hasseldam H, Bozyczko-Coyne D, Morath S, Hartung T, Bianchi M, Ghezzi P, Bsibsi M et al. Inhibition of microglial inflammation by the MLK inhibitor CEP-1347. J Neurochem. 2005;92(6):1439-51.

13. Horvath RJ, Nutile-McMenemy N, Alkaitis MS, Deleo JA. Differential migration, LPS-induced cytokine, chemokine, and NO expression in immortalized BV-2 and HAPI cell lines and primary microglial cultures. J Neurochem. 2008;107(2):557-69.

14. Butovsky O, Jedrychowski MP, Moore CS, Cialic R, Lanser AJ, Gabriely G, Koeglsperger T, Dake B, Wu PM, Doykan CE et al. Identification of a unique TGF-beta-dependent molecular and functional signature in microglia. Nat Neurosci. 2014;17(1):131-43.

15. Stansley B, Post J, Hensley K. A comparative review of cell culture systems for the study of microglial biology in Alzheimer's disease. J Neuroinflammation. 2012;9:115.

16. Lund S, Christensen KV, Hedtjarn M, Mortensen AL, Hagberg H, Falsig J, Hasseldam H, Schrattenholz A, Porzgen P, Leist M. The dynamics of the LPS triggered inflammatory response of murine microglia under different culture and in vivo conditions. J Neuroimmunol. 2006;180(1-2):71-87.

17. Sultan M, Schulz MH, Richard H, Magen A, Klingenhoff A, Scherf M, Seifert $M$, Borodina T, Soldatov A, Parkhomchuk D, et al. A global view of gene activity and alternative splicing by deep sequencing of the human transcriptome. Science. 2008;321(5891):956-60.

18. Nagalakshmi U, Wang Z, Waern K, Shou C, Raha D, Gerstein M, Snyder M. The transcriptional landscape of the yeast genome defined by RNA sequencing. Science. 2008;320(5881):1344-9.

19. Witting A, Moller T. Microglia cell culture: a primer for the novice. Methods Mol Biol. 2011;758:49-66.

20. Das A, Chai JC, Kim SH, Park KS, Lee YS, Jung KH, Chai YG. Dual RNA sequencing reveals the expression of unique transcriptomic signatures in lipopolysaccharide-induced BV-2 microglial cells. PLoS One. 2015;10(3):e0121117
21. Thomas DM, Francescutti-Verbeem DM, Kuhn DM. Gene expression profile of activated microglia under conditions associated with dopamine neuronal damage. FASEB journal: official publication of the Federation of American Societies for Experimental Biology. 2006;20(3):515-7.

22. Bolger AM, Lohse M, Usadel B. Trimmomatic: a flexible trimmer for Illumina sequence data. Bioinformatics. 2014;30(15):2114-20.

23. Dobin A, Davis CA, Schlesinger F, Drenkow J, Zaleski C, Jha S, Batut P, Chaisson M, Gingeras TR. STAR: ultrafast universal RNA-seq aligner. Bioinformatics. 2013;29(1):15-21.

24. Love MI, Huber W, Anders S. Moderated estimation of fold change and dispersion for RNA-seq data with DESeq2. Genome Biol. 2014;15(12):550

25. Heinz S, Benner C, Spann N, Bertolino E, Lin YC, Laslo P, Cheng JX, Murre C, Singh $\mathrm{H}$, Glass CK. Simple combinations of lineage-determining transcription factors prime cis-regulatory elements required for macrophage and B cell identities. Mol Cell. 2010;38(4):576-89.

26. Jung KH, Das A, Chai JC, Kim SH, Morya N, Park KS, Lee YS, Chai YG. RNA sequencing reveals distinct mechanisms underlying BET inhibitor JQ1mediated modulation of the LPS-induced activation of BV-2 microglial cells. J Neuroinflammation. 2015;12:36.

27. da Huang W, Sherman BT, Lempicki RA. Systematic and integrative analysis of large gene lists using DAVID bioinformatics resources. Nat Protoc 2009;4(1):44-57.

28. Kramer A, Green J, Pollard Jr J, Tugendreich S. Causal analysis approaches in Ingenuity Pathway Analysis. Bioinformatics. 2014;30(4):523-30.

29. Zambelli F, Pesole G, Pavesi G. Pscan: finding over-represented transcription factor binding site motifs in sequences from co-regulated or co-expressed genes. Nucleic Acids Res. 2009;37(Web Server issue):W247-252.

30. Portales-Casamar E, Thongjuea S, Kwon AT, Arenillas D, Zhao X, Valen E, Yusuf D, Lenhard B, Wasserman WW, Sandelin A. JASPAR 2010: the greatly expanded open-access database of transcription factor binding profiles. Nucleic Acids Res. 2010;38(Database issue):D105-110.

31. Das A, Chai JC, Kim SH, Lee YS, Park KS, Jung KH, Chai YG. Transcriptome sequencing of microglial cells stimulated with TLR3 and TLR4 ligands. BMC Genomics. 2015;16:517.

32. Dijkstra IM, de Haas AH, Brouwer N, Boddeke HW, Biber K. Challenge with innate and protein antigens induces CCR7 expression by microglia in vitro and in vivo. Glia. 2006;54(8):861-72.

33. Borden EC, Sen GC, Uze G, Silverman RH, Ransohoff RM, Foster GR, Stark GR. Interferons at age 50: past, current and future impact on biomedicine. Nat Rev Drug Discov. 2007:6(12):975-90.

34. Shoemaker ML, Smirnova NP, Bielefeldt-Ohmann H, Austin KJ, van Olphen A, Clapper JA, Hansen TR. Differential expression of the type I interferon pathway during persistent and transient bovine viral diarrhea virus infection. Journal of interferon \& cytokine research: the official journal of the International Society for Interferon and Cytokine Research. 2009;29(1):23-35.

35. De Jesus AF, Chen G, Li Z, Grayfer L, Robert J. Susceptibility of Xenopus laevis tadpoles to infection by the ranavirus Frog-Virus 3 correlates with a reduced and delayed innate immune response in comparison with adult frogs. Virology. 2012;432(2):435-43.

36. O'Shea JJ, Plenge R. JAK and STAT signaling molecules in immunoregulation and immune-mediated disease. Immunity. 2012;36(4):542-50.

37. Forero A, Giacobbi NS, McCormick KD, Gjoerup OV, Bakkenist CJ, Pipas JM, Sarkar SN. Simian virus 40 large T antigen induces IFN-stimulated genes through ATR kinase. J Immunol. 2014;192(12):5933-42.

38. Andreoli F, Barbosa AJ, Parenti MD, Del Rio A. Modulation of epigenetic targets for anticancer therapy: clinicopathological relevance, structural data and drug discovery perspectives. Curr Pharm Des. 2013;19(4):578-613.

39. Schliehe C, Flynn EK, Vilagos B, Richson U, Swaminathan S, Bosnjak B, Bauer L, Kandasamy RK, Griesshammer IM, Kosack L et al. The methyltransferase Setdb2 mediates virus-induced susceptibility to bacterial superinfection. Nat Immunol. 2015;16(1):67-74.

40. Ponomarev ED, Veremeyko T, Barteneva N, Krichevsky AM, Weiner HL. MicroRNA124 promotes microglia quiescence and suppresses EAE by deactivating macrophages via the C/EBP-alpha-PU.1 pathway. Nat Med. 2011;17(1):64-70.

41. Cao M, Cortes M, Moore CS, Leong SY, Durosier LD, Burns P, Fecteau G, Desrochers A, Auer RN, Barreiro LB et al. Fetal microglial phenotype in vitro carries memory of prior in vivo exposure to inflammation. Front Cell Neurosci. 2015;9:294.

42. Takeshita Y, Ransohoff RM. Inflammatory cell trafficking across the blood-brain barrier: chemokine regulation and in vitro models. Immunol Rev. 2012;248(1):228-39. 
43. Constantinescu CS, Goodman DB, Hilliard B, Wysocka M, Cohen JA. Murine macrophages stimulated with central and peripheral nervous system myelin or purified myelin proteins release inflammatory products. Neurosci Lett. 2000;287(3):171-4.

44. Zhang Z, Li LY. TNFSF15 modulates neovascularization and inflammation. Cancer microenvironment: official journal of the International Cancer Microenvironment Society. 2012;5(3):237-47.

45. Proudfoot AE, de Souza AL, Muzio V. The use of chemokine antagonists in EAE models. J Neuroimmunol. 2008;198(1-2):27-30.

46. Si Q, Kim MO, Zhao ML, Landau NR, Goldstein H, Lee S. Vpr- and Nefdependent induction of RANTES/CCL5 in microglial cells. Virology. 2002; 301(2):342-53.

47. Lundberg AM, Drexler SK, Monaco C, Williams LM, Sacre SM, Feldmann M, Foxwell BM. Key differences in TLR3/poly I:C signaling and cytokine induction by human primary cells: a phenomenon absent from murine cell systems. Blood. 2007;110(9):3245-52.

48. Williams R, Dhillon NK, Hegde ST, Yao H, Peng F, Callen S, Chebloune Y, Davis RL, Buch SJ. Proinflammatory cytokines and HIV-1 synergistically enhance CXCL10 expression in human astrocytes. Glia. 2009;57(7):734-43.

49. Shen $\mathrm{Q}$, Zhang R, Bhat NR. MAP kinase regulation of IP10/CXCL10 chemokine gene expression in microglial cells. Brain Res. 2006;1086(1):9-16.

50. Lazear HM, Lancaster A, Wilkins C, Suthar MS, Huang A, Vick SC, Clepper L, Thackray L, Brassil MM, Virgin HW et al. IRF-3, IRF-5, and IRF-7 coordinately regulate the type I IFN response in myeloid dendritic cells downstream of MAVS signaling. PLoS Pathog. 2013;9(1):e1003118.

51. Honda K, Yanai H, Negishi H, Asagiri M, Sato M, Mizutani T, Shimada N, Ohba Y, Takaoka A, Yoshida N et al. IRF-7 is the master regulator of type-I interferon-dependent immune responses. Nature. 2005; 434(7034):772-7.

52. Inoue M, Williams KL, Gunn MD, Shinohara ML. NLRP3 inflammasome induces chemotactic immune cell migration to the CNS in experimental autoimmune encephalomyelitis. Proc Natl Acad Sci U S A. 2012;109(26):10480-5.

53. Martinon F, Tschopp J. Inflammatory caspases and inflammasomes: master switches of inflammation. Cell Death Differ. 2007;14(1):10-22.

54. Xiao Y, Jin J, Chang M, Chang JH, Hu H, Zhou X, Brittain GC, Stansberg C, Torkildsen O, Wang X. Peli1 promotes microglia-mediated CNS inflammation by regulating Traf3 degradation. Nat Med. 2013;19(5):595-602.

55. Smith AM, Gibbons HM, Oldfield RL, Bergin PM, Mee EW, Curtis MA, Faull RL, Dragunow M. M-CSF increases proliferation and phagocytosis while modulating receptor and transcription factor expression in adult human microglia. J Neuroinflammation. 2013;10:85.

56. Melief J, Koning N, Schuurman KG, Van De Garde MD, Smolders J, Hoek RM, Van Eijk M, Hamann J, Huitinga I. Phenotyping primary human microglia: tight regulation of LPS responsiveness. Glia. 2012;60(10):1506-17.

57. Lai AY, Dibal CD, Armitage GA, Winship IR, Todd KG. Distinct activation profiles in microglia of different ages: a systematic study in isolated embryonic to aged microglial cultures. Neuroscience. 2013;254:185-95.

58. Njie EG, Boelen E, Stassen FR, Steinbusch HW, Borchelt DR, Streit WJ. Ex vivo cultures of microglia from young and aged rodent brain reveal age-related changes in microglial function. Neurobiol Aging. 2012;33(1):195 e191-112.

59. Hanisch UK. Functional diversity of microglia-how heterogeneous are they to begin with? Front Cell Neurosci. 2013;7:65.

60. Hanisch UK, Kettenmann H. Microglia: active sensor and versatile effector cells in the normal and pathologic brain. Nat Neurosci. 2007;10(11):1387-94.

61. Tang $Y$, Le $W$. Differential roles of $M 1$ and $M 2$ microglia in neurodegenerative diseases. Mol Neurobiol. 2016;53(2):1181-94.

62. Kitamura Y, Taniguchi T, Kimura H, Nomura Y, Gebicke-Haerter PJ. Interleukin-4-inhibited mRNA expression in mixed rat glial and in isolated microglial cultures. J Neuroimmunol. 2000;106(1-2):95-104.

63. Ledeboer A, Breve JJ, Poole S, Tilders FJ, Van Dam AM. Interleukin-10, interleukin-4, and transforming growth factor-beta differentially regulate lipopolysaccharide-induced production of pro-inflammatory cytokines and nitric oxide in co-cultures of rat astroglial and microglial cells. Glia. 2000;30(2):134-42.

64. Siddiqui TA, Lively S, Schlichter LC. Complex molecular and functional outcomes of single versus sequential cytokine stimulation of rat microglia. J Neuroinflammation. 2016;13(1):66.

65. Dohi K, Ohtaki H, Nakamachi T, Yofu S, Satoh K, Miyamoto K, Song D, Tsunawaki S, Shioda S, Aruga T. Gp91phox (NOX2) in classically activated microglia exacerbates traumatic brain injury. J Neuroinflammation. 2010;7:41.
66. Ghosh M, Xu Y, Pearse DD. Cyclic AMP is a key regulator of M1 to M2a phenotypic conversion of microglia in the presence of Th2 cytokines. J Neuroinflammation. 2016;13:9.

67. Gandy S, Heppner FL. Microglia as dynamic and essential components of the amyloid hypothesis. Neuron. 2013;78(4):575-7.

68. Perry VH, Holmes C. Microglial priming in neurodegenerative disease. Nat Rev Neurol. 2014;10(4):217-24.

69. Shimizu E, Kawahara K, Kajizono M, Sawada M, Nakayama H. IL-4-induced selective clearance of oligomeric beta-amyloid peptide(1-42) by rat primary type 2 microglia. J Immunol. 2008;181(9):6503-13.

\section{Submit your next manuscript to BioMed Central and we will help you at every step:}

- We accept pre-submission inquiries

- Our selector tool helps you to find the most relevant journal

- We provide round the clock customer support

- Convenient online submission

- Thorough peer review

- Inclusion in PubMed and all major indexing services

- Maximum visibility for your research

Submit your manuscript at www.biomedcentral.com/submit
Biomed Central 